\title{
sEMG-MMG State-Space Model for the Continuous Estimation of Multijoint Angle
}

\author{
Xugang Xi $\mathbb{D}^{1,2}$ Chen Yang, ${ }^{1,2}$ Seyed M. Miran, ${ }^{3}$ Yun-Bo Zhao ${ }^{1},{ }^{4}$ Shuliang Lin, ${ }^{5}$ \\ and Zhizeng Luo $\mathbb{D}^{1,2}$ \\ ${ }^{1}$ School of Artificial Intelligence, Hangzhou Dianzi University, Hangzhou 310018, China \\ ${ }^{2}$ Key Laboratory of Brain Machine Collaborative Intelligence of Zhejiang Province, Hangzhou 310018, China \\ ${ }^{3}$ Biomedical Informatics Center, George Washington University, Washington, DC 20052, USA \\ ${ }^{4}$ Department of Automation, Zhejiang University of Technology, Hangzhou 310023, China \\ ${ }^{5}$ Jinhua Municipal Central Hospital, Jinhua 321000, China \\ Correspondence should be addressed to Xugang Xi; xixi@hdu.edu.cn and Zhizeng Luo; luo@hdu.edu.cn
}

Received 18 July 2019; Revised 14 December 2019; Accepted 16 January 2020; Published 11 February 2020

Guest Editor: Gonzalo Gutiérrez-Tobal

Copyright ( $\odot 2020$ Xugang Xi et al. This is an open access article distributed under the Creative Commons Attribution License, which permits unrestricted use, distribution, and reproduction in any medium, provided the original work is properly cited.

\begin{abstract}
Continuous joint angle estimation plays an important role in motion intention recognition and rehabilitation training. In this study, a surface electromyography- (sEMG-) mechanomyography (MMG) state-space model is proposed to estimate continuous multijoint movements from sEMG and MMG signals accurately. The model combines forward dynamics with a Hill-based muscle model that estimates joint torque only in a nonfeedback form, making the extended model capable of predicting the multijoint motion directly. The sEMG and MMG features, including the Wilson amplitude and permutation entropy, are then extracted to construct a measurement equation to reduce system error and external disturbances. Using the proposed model, a closed-loop prediction-correction approach, unscented particle filtering, is used to estimate the joint angle from sEMG and MMG signals. Comprehensive experiments are conducted on the human elbow and shoulder joint, and remarkable improvements are demonstrated compared with conventional methods.
\end{abstract}

\section{Introduction}

In human-machine interaction (HMI), surface electromyography (sEMG) is often used to serve as the input signal source. The sEMG signal is a weak electrical potential generated by muscle cells upon electrical or neurological activation, and it is detected from superficial muscles by using surface electrodes. The signal contains abundant information and has a distinct characteristic. Moreover, its collection is simple and not traumatic. The sEMG signal has become a research hotspot in the field of human-computer interaction technology, especially in the manufacturing of exoskeleton robots [1], intelligent prosthetics [2], and rehabilitation robots [3].

sEMG studies usually focus on feature extraction and pattern classification. Identifying a plurality of discrete action categories has an accuracy rate of approximately 90\% [4].
Studies on the classification of discrete actions have been done from the laboratory to the market [5]. Estimating continuous human joint movement is a critical focus in sEMG at present. Many achievements have already been made, especially in the field of rehabilitation robots, where forecasting the continuous motion variables of patients is vital to achieve smooth control of the rehabilitation robot [6]. However, the sEMG signal is susceptible to interference from sweat and skin impedance, which consequently deteriorates the control accuracy.

Mechanomyography (MMG) is a mechanical signal produced by contracting muscles, and it reflects muscle activity in the form of low-frequency $(<50 \mathrm{~Hz})$ vibration. In contrast to sEMG signals, MMG signals are unaffected by sweat and changes in skin impedance. Recent studies have proven the efficacy of MMG, such as in upper-limb prostheses control [7-9] and hand movement analysis [10-13]. 
However, the MMG signal has considerably lower signal-tonoise ratios than the sEMG signal. External noise caused by movement artifact can also introduce interference to MMG measurement. Thus, combining the advantages of both sEMG and MMG would be of great significance to understanding muscle activities and enhancing HMI.

Extracting features such as root mean square and the absolute value of amplitude and then establishing a motion model with neural networks constitute an effective method for estimating continuous joint motion $[14,15]$. The estimation can also be performed through the physiological muscle model, for which the Hill-based muscle model (HMM) is often used [16, 17]. However, the HMM presents two challenges: (1) the HMM involves many complex parameters that are difficult to identify and is computationally heavy, and (2) in the HMM, motion states are indirectly calculated from the sEMG and MMG signals, thereby generating accumulative errors and worsening the estimation accuracy.

To address the above challenges, this study developed a new model that fuses sEMG and MMG signals and brings together HMM and joint dynamics. This model can directly calculate the joint motion from sEMG and MMG signals. Wilson amplitude (WA) and permutation entropy (PE) are extracted to establish the measurement equations. Then, an sEMG-MMG state-space model is created to estimate the mutijoint angle.

\section{Methodology}

As shown in Figure 1, the sEMG-MMG state-space model consists of three parts: (1) the sEMG and MMG signals to the joint forward dynamics, (2) the fusion of sEMG and MMG features, and (3) the prediction algorithm. The model aims to estimate the joint moving states through the muscle model and fusion features.

2.1. Data Acquisition. The muscles relevant to elbow and shoulder joint motion were selected, including the biceps brachii (BB), triceps brachii (TB), brachioradialis (BR), trapezius (TZ), teres minor (TM), anterior deltoid (AD), lateral deltoid (LD), and pectoralis major (PM). The sEMG and MMG signals were measured by placing eight surface electrodes on the muscles of the right upper limb (Figure 2).

The Trigno ${ }^{\mathrm{TM}}$ Wireless (Delsys Inc, Natick, MA, USA) was used to record the sEMG and MMG signals at $1600 \mathrm{~Hz}$. The bandwidth was $20-450 \mathrm{~Hz}$, and the baseline noise was less than $1.25 \mu \mathrm{V}$. The sensors were positioned following the SENIAM recommendation. The reference electrode was attached to the lateral elbow epicondyle.

The Codamotion system (Charnwood Dynamics Ltd., UK) was used to record the real joint angle information. This system can capture and analyze motion information in real time through infrared capture. Figure 3 shows the Codamotion system and placement of markers.

Ten healthy subjects (five males and five females, $23 \pm 2$ years old, weighing $50-70 \mathrm{~kg}$ ) were tested in the experiments. The study was approved by the Hangzhou Dianzi
University's Institutional Review Board, and all subjects provided a written informed consent prior to participation. Figure 4 shows the experimental movements. The subject (1) sits on a chair and keeps the muscles relaxed, (2) straightens the right arm forward with elbow extension, (3) performs elbow flexion, (4) straightens the arm upward with elbow extension, (5) straightens the arm to the right side with elbow extension, (6) performs elbow flexion, and (7) returns the upper arm to the initial position while maintaining elbow flexion. The experimental movements involved the main activities of the upper limb, thereby enabling us to better analyze the movement of the shoulder and elbow joints. Each subject completed all movements with steps 1-2-3-4-5-6-7-1 in $15 \mathrm{~s}$ and performed the sequence with or without a load $(3 \mathrm{~kg})$. The subjects were given an appropriate rest time after each round to reduce muscle fatigue. We completed the data collection within five days, and the subjects repeated the procedure 10 times in each experiment day. In this way, we were able to collect the required data.

2.2. Data Preprocessing. Both sEMG and MMG signals are very weak, nonstationary, and random. Many kinds of noise exist in the acquisition process. The sEMG and MMG signals must be denoised for further application. In this experiment, the Trigno Wireless (Delsys Inc.) with a bandpass filter (sEMG and MMG signals of $20-450$ and $5-150 \mathrm{~Hz}$, respectively) was used to eliminate most of the inherent noises and motion artifacts.

The sampling frequency of the joint angle signal is $100 \mathrm{~Hz}$, and that of the sEMG and MMG signals is $1600 \mathrm{~Hz}$. To keep the sEMG and MMG signals consistent with the joint angle signal, subsampling of the sEMG signals was implemented using feature extraction. Afterward, the subsampling frequency of the sEMG and MMG signals was changed from $1600 \mathrm{~Hz}$ to $100 \mathrm{~Hz}$.

\section{3. sEMG-MMG State-Space Model}

2.3.1. Hill-Based Muscle Model. Figure 5 illustrates a simplified HMM. The HMM consists of a contractile element and a parallel elastic element. The contractile element produces the active muscle force $F_{A}^{m}$, and the parallel elastic element produces the passive force $F_{P}^{m}$. The model can be described as follows:

$$
\begin{aligned}
F^{m} & =F_{P}^{m}+F_{A}^{m}=f_{P}(l) \cdot F_{0}^{m}+f_{A}(l) \cdot f_{V}(v) \cdot a(k) \cdot F_{0}^{m}, \\
l & =\frac{l^{m}}{l_{0}^{m}} \\
v & =\frac{v^{m}}{v_{0}^{m}}
\end{aligned}
$$

where $F^{\mathrm{mt}}$ is the musculotendon force, $f_{P}(l)$ is the normalized passive force-length relationship, $F_{0}^{m}$ is the maximum isometric force, $l$ is the normalized muscle fiber length, $l^{m}$ is the current muscle fiber length, $l_{0}^{m}$ is the optimal fiber 


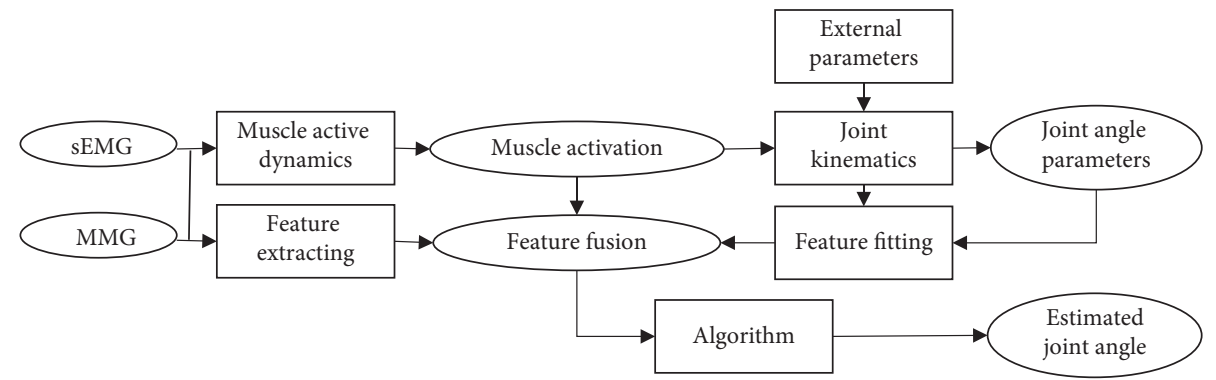

Figure 1: Structure of the sEMG-MMG state-space model. (1) The sEMG and MMG signals to the joint forward dynamics, (2) the fusion of sEMG and MMG features, and (3) the prediction algorithm.
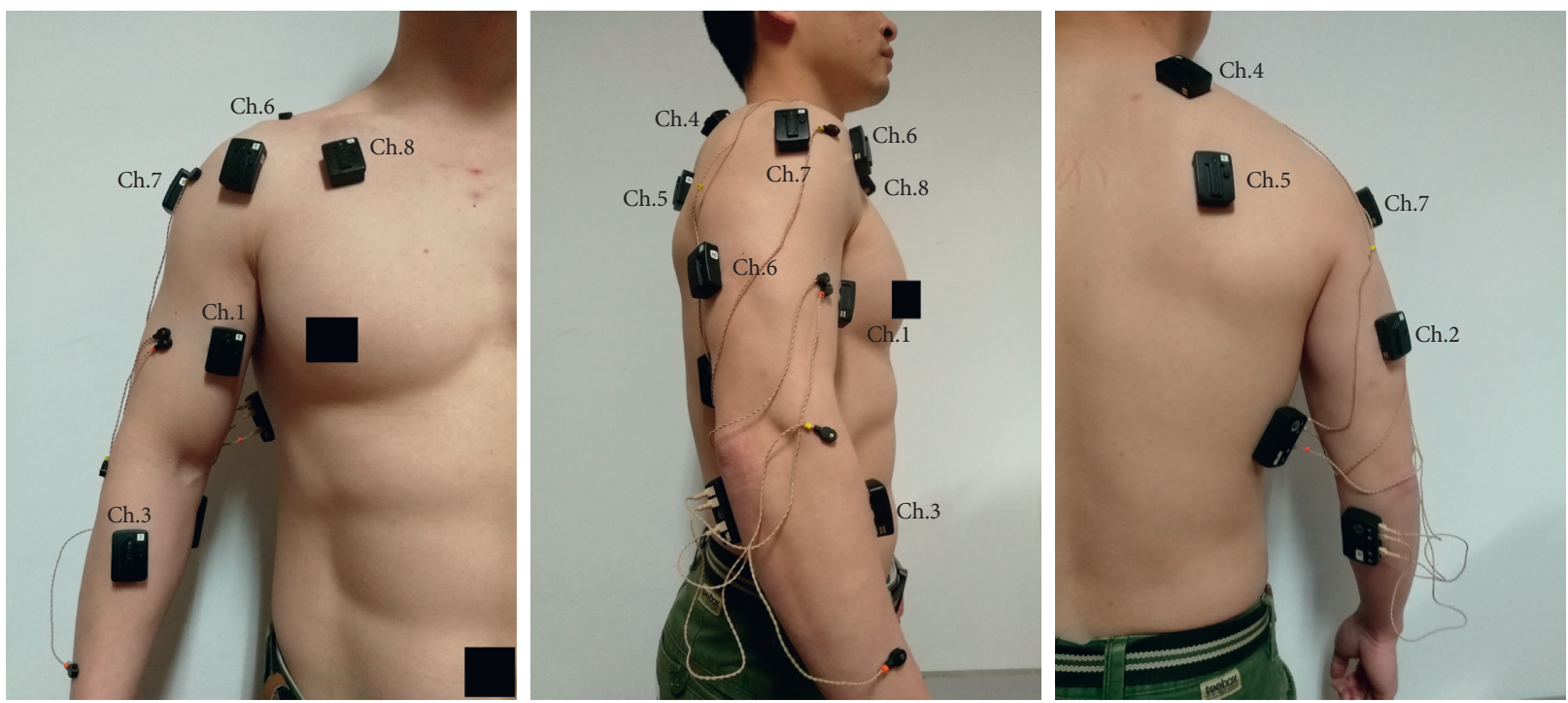

FIgure 2: sEMG sensor arrangement (Ch1: BB, Ch2: TB, Ch3: BR, Ch4: TZ, Ch5: TM, Ch6: AD, Ch7: LD, and Ch8: PM).
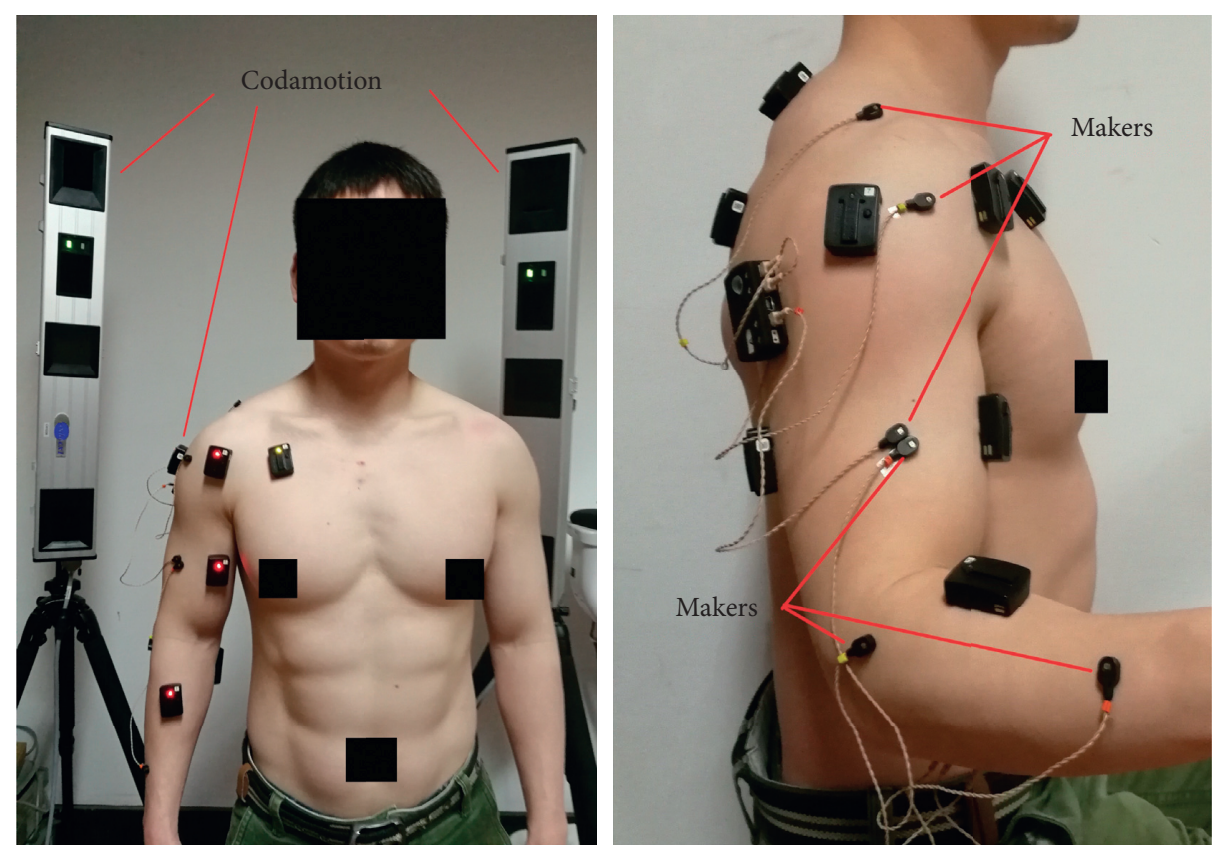

Figure 3: Codamotion system and placement of markers. 


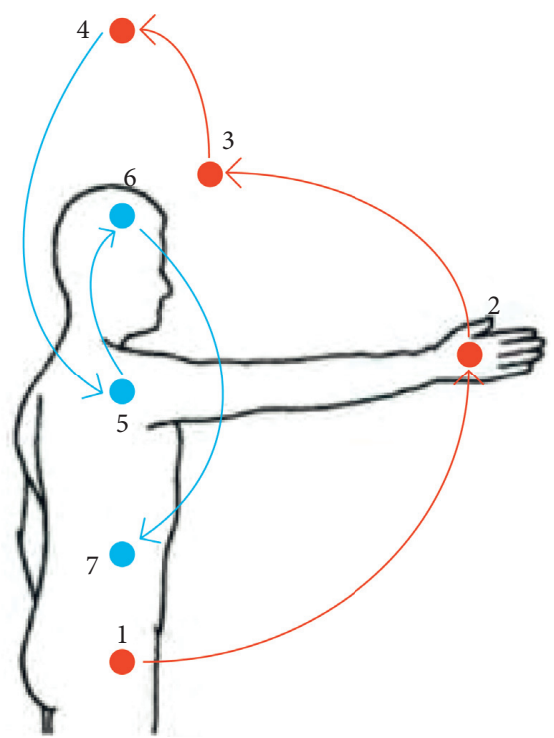

(a)

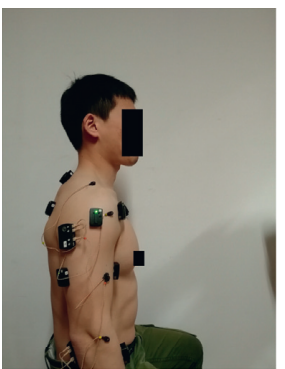

Step 1

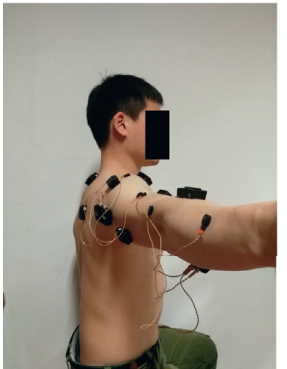

Step 5

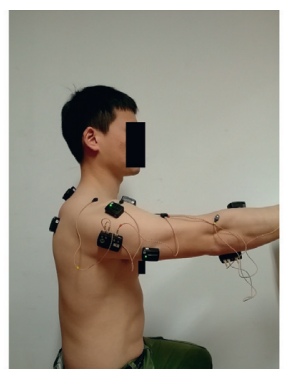

Step 2

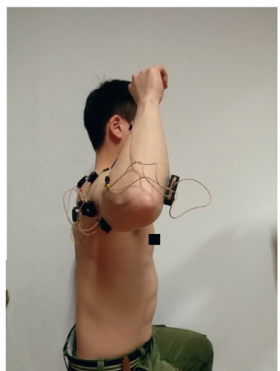

Step 6

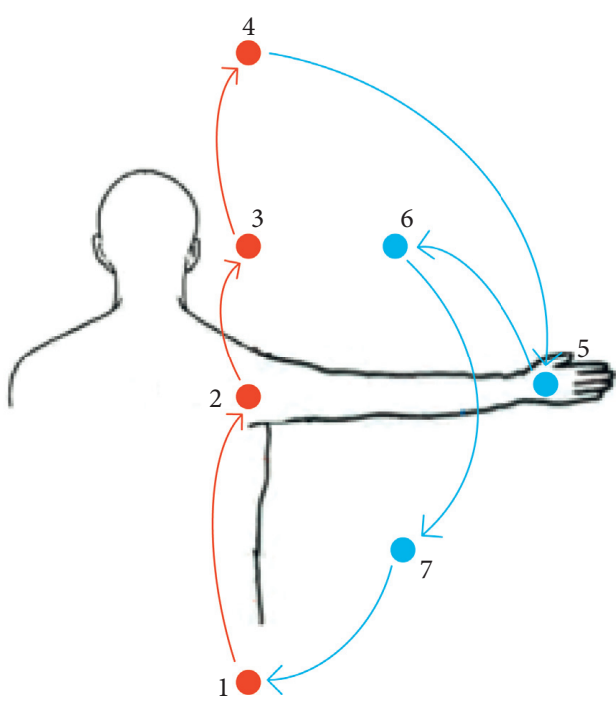

(b)

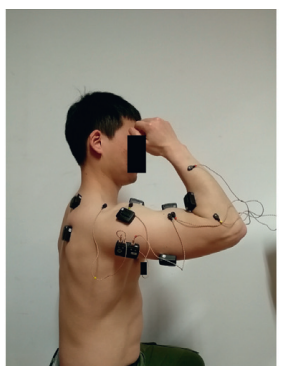

Step 3

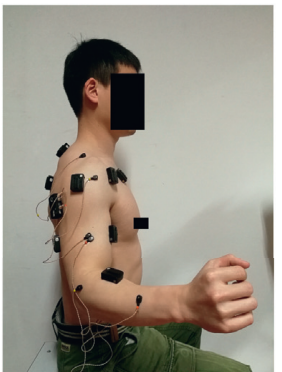

Step 7

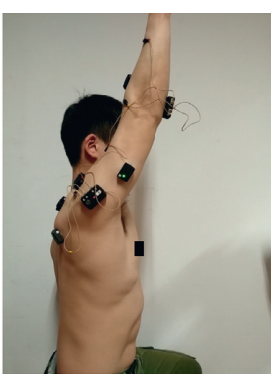

Step 4

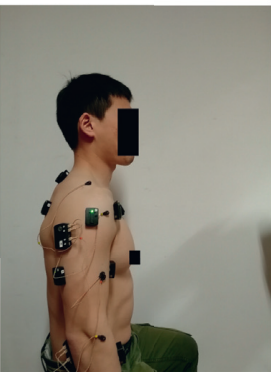

Step 1

Figure 4: Upper limb movements recorded: steps 1-2-3-4-5-6-7-1. Schematic of the (a) right-hand side and (b) back side.

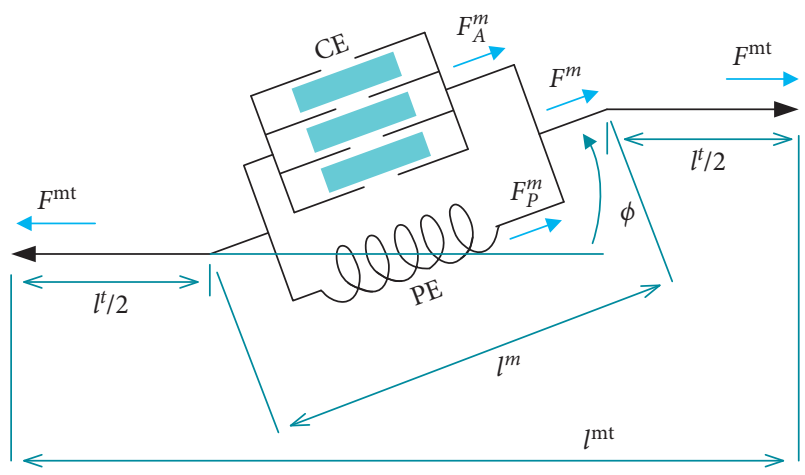

Figure 5: Hill-based muscle model. length, $f_{A}(l)$ is the normalized active force-length relationship, $f_{V}(v)$ is the normalized force-velocity relationship, $v$ is the normalized muscle fiber velocity, $v^{m}$ is the muscle contraction velocity, $v_{0}^{m}$ is the maximum muscle contraction velocity, and $a(k)$ is the muscle activation [18].

According to the abovementioned equations, the musculotendon force can be described as follows:

$$
F^{\mathrm{mt}}=\left[f_{A}(l) \cdot f_{V}(v) \cdot a(k)+f_{P}(l)\right] \cdot F_{0}^{m} \cdot \cos (\phi),
$$

where $F^{\mathrm{mt}}$ is the musculotendon force and $\phi$ is the pennation angle.

The simplifications of $f_{A}(l), f_{V}(v), f_{P}(l), F_{0}^{m}, l^{t}, l_{0}^{m}$, and $\phi$ can replace the complex biomechanical parameters [19-22]. 
The musculotendon length can also be simplified as a firstorder polynomial $[23,24]$. Thus,

$$
l^{\mathrm{mt}}=b_{0}+b_{1} \cdot \theta
$$

where $l^{\mathrm{mt}}$ is the musculotendon length, $b_{0}$ and $b_{1}$ are constants, and $\theta$ is the joint angle. Then, the moment arm $r$ and the joint moment $\tau$ can be calculated as follows:

$$
\begin{aligned}
& r=\frac{\partial l^{\mathrm{mt}}(\theta)}{\partial \theta}=b_{1}, \\
& \tau=F^{\mathrm{mt}} \cdot r .
\end{aligned}
$$

2.3.2. sEMG-MMG Joint Motion Model. The angular acceleration of the joint can be calculated as follows:

$$
\ddot{\theta}=\frac{1}{I_{e}} \cdot\left(\tau-\tau_{\mathrm{eg}}\right), \tau_{\mathrm{eg}}=\tau_{\mathrm{egm}} \cdot \sin (\theta),
$$

where $I_{e}$ is the moment of inertia, $\tau_{\text {eg }}$ is the external and gravity torque, and $\tau_{\text {egm }}$ is the maximum of $\tau_{\text {eg }}$.

By combining the above equations, the angular acceleration $\ddot{\theta}$ can be described as follows:

$$
\ddot{\theta}=\left(s_{0}+s_{1} \cdot \theta+s_{2} \cdot \theta^{2}\right) \cdot a(k)+s_{3} \cdot e^{s_{4} \cdot \theta}-s_{5} \cdot \sin (\theta),
$$

where $s_{i}(i=0,1, \ldots, 5)$ are constants computed as follows:

$$
\left\{\begin{array}{l}
s_{0}=\frac{k_{0} \cdot F_{0}^{m} \cdot b_{1} \cdot \cos \phi}{I_{e}}+\frac{k_{1} \cdot F_{0}^{m} \cdot\left(b_{0}-l^{t}\right) \cdot b_{1}}{l_{0}^{m} \cdot I_{e}}+\frac{k_{2} \cdot F_{0}^{m} \cdot\left(b_{0}-l^{t}\right)^{2} \cdot b_{1}}{\left(l_{0}^{m}\right)^{2} \cdot I_{e} \cdot \cos \phi}, \\
s_{1}=\frac{k_{1} \cdot F_{0}^{m} \cdot\left(b_{1}\right)^{2}}{l_{0}^{m} \cdot I_{e}}+\frac{2 \cdot k_{2} \cdot\left(b_{0}-l^{t}\right) \cdot\left(b_{1}\right)^{2}}{\left(l_{0}^{m}\right)^{2} \cdot I_{e} \cdot \cos \phi}, \\
s_{2}=\frac{k_{2} \cdot F_{0}^{m} \cdot\left(b_{1}\right)^{3}}{\left(l_{0}^{m}\right)^{2} \cdot I_{e} \cdot \cos \phi}, \\
s_{3}=\frac{F_{0}^{m} \cdot b_{1} \cdot \cos \phi}{I_{e}} \cdot \exp \left(\frac{10 \cdot\left(b_{0}-l^{t}\right) \cdot\left(b_{1}\right)^{2}}{l_{0}^{m} \cdot \cos \phi}-15\right), \\
s_{4}=\frac{10 \cdot b_{1}}{l_{0}^{m} \cdot \cos \phi}, \\
s_{5}=\frac{\tau_{\text {egm }}}{I_{e}} .
\end{array}\right.
$$

Then, we can obtain the sEMG-MMG joint motion model in discrete time as follows:

$$
\left\{\begin{array}{l}
\ddot{\theta}_{k+1}=\left(s_{0}+s_{1} \cdot \theta_{k}+s_{2} \cdot \theta_{k}^{2}\right) \cdot a(k)+s_{3} \cdot e^{s_{4} \cdot \theta_{k}}-s_{5} \cdot \sin \left(\theta_{k}\right), \\
\dot{\theta}_{k+1}=\dot{\theta}_{k}+\ddot{\theta}_{k} \cdot T_{s}, \\
\theta_{k+1}=\theta_{k}+\dot{\theta}_{k} \cdot T_{s},
\end{array}\right.
$$

where $\dot{\theta}_{k}$ is the joint angular velocity, $\theta_{k}$ is the joint position, and $T_{s}$ is the sampling time.

2.3.3. sEMG-MMG Features and State-Space Model. The simplified sEMG-MMG joint motion model involves two problems. First, the model omits certain physiological parameters. Second, the biomechanical parameters vary because of different body conditions. These problems cause accumulative errors in the recursive calculation of the joint angle. To eliminate these errors, we constructed a measurement equation providing the joint angle measurements as feedback, which then leads to a sEMG-MMG state-space model. We built the measure equation by Wilson amplitude (WA) and permutation entropy (PE) in this study.

(1) WA:

The WA describes the different characteristics among adjacent amplitudes [25]. It can be described as follows:

$$
\mathrm{WA}=\sum_{i=1}^{N} u\left(\left|x_{i+1}-x_{i}-T\right|\right),
$$

where $x_{i}$ and $x_{i+1}$ are adjacent samples, and the threshold $T$ is $0.05 \mathrm{~V}$ in this study.

(2) PE:

The PE describes the complexity of the data and has the advantages of good robustness and high antiinterference $[26,27]$. It can be described as follows: 


$$
H(n)=-\sum_{\pi=1}^{n !} p(\pi) \ln (p(\pi))
$$

where $p(\pi)$ is the probability statistics in the entire time series, $n$ is an $n$-dimensional vector making, and $\pi$ is the different permutation ways.

To relate the sEMG and MMG features with the joint motion model, the following second-order polynomial was used as the fitting function:

$$
y_{k}^{u}=c_{0}^{u}+c_{1}^{u} \cdot \dot{\theta}_{k}+c_{2}^{u} \cdot \theta_{k}+c_{3}^{u} \cdot \dot{\theta}_{k}^{2}+c_{4}^{u} \cdot \theta_{k}^{2}+c_{5}^{u} \cdot \dot{\theta}_{k} \cdot \theta_{k} \text {, }
$$

where $u=1,2, c_{i}^{u}(i=0,1, \ldots, 5)$ are constant parameters, $y_{k}^{1}$ is the WA of the sEMG and MMG signals, and $y_{k}^{2}$ is the PE of sEMG and MMG signals.

By combining the equations above, the nonlinear expression of the model was obtained:

$$
\begin{gathered}
\left\{\begin{array}{l}
x_{k+1}=f\left(x_{k}, a_{k}\right)+\omega_{k}, \\
y_{k+1}=h\left(x_{k+1}\right)+v_{k+1},
\end{array}\right. \\
f\left(x_{k}, a_{k}\right)=\left[\begin{array}{c}
\left(s_{0}+s_{1} \cdot \theta_{k}+s_{2} \cdot \theta_{k}^{2}\right) \cdot a_{k}+s_{3} \cdot e^{s_{4} \cdot \theta_{k}}-s_{5} \cdot \sin \left(\theta_{k}\right) \\
\dot{\theta}_{k}+\ddot{\theta}_{k} \cdot T \\
\theta_{k}+\dot{\theta}_{k} \cdot T
\end{array}\right], \\
h\left(x_{k}\right)=\left[\begin{array}{c}
c_{0}^{1}+c_{1}^{1} \cdot \dot{\theta}_{k}+c_{2}^{1} \cdot \theta_{k}+c_{3}^{1} \cdot \dot{\theta}_{k}^{2}+c_{4}^{1} \cdot \theta_{k}^{2}+c_{5}^{1} \cdot \dot{\theta}_{k} \cdot \theta_{k} \\
c_{0}^{2}+c_{1}^{2} \cdot \dot{\theta}_{k}+c_{2}^{2} \cdot \theta_{k}+c_{3}^{2} \cdot \dot{\theta}_{k}^{2}+c_{4}^{2} \cdot \theta_{k}^{2}+c_{5}^{2} \cdot \dot{\theta}_{k} \cdot \theta_{k}
\end{array}\right],
\end{gathered}
$$

where $\omega_{k}$ is the process noise, $v_{k}$ is the measurement noise, $a_{k}$ represents the muscle activation $a(k), x_{k}=\left[\begin{array}{lll}\ddot{\theta}_{k} & \dot{\theta}_{k} & \theta_{k}\end{array}\right]^{T}$, and $y_{k}=\left[\begin{array}{ll}y_{k}^{1} & y_{k}^{2}\end{array}\right]^{T}$.

2.3.4. Unscented Particle Filtering (UPF). The UPF consists of the generic particle filter (PF) and the unscented Kalman filter (UKF). The UPF first uses the UKF to generate the proposal distribution for the $\mathrm{PF}$, and the rest of the steps are the same as PF [28]. The steps to generate the proposal distribution using UKF are as follows:

Particles are updated with the UKF using the following equations:

$$
\begin{aligned}
X_{0} & =\bar{x} \\
X_{i} & =\bar{x}+\left(\sqrt{\left(n_{x}+\lambda\right) P_{x}}\right)_{i} \quad\left(i=1, \ldots, n_{x}\right) \\
X_{i} & =\bar{x}-\left(\sqrt{\left(n_{x}+\lambda\right) P_{x}}\right)_{i} \quad\left(i=n_{x}, \ldots, 2 n_{x}\right), \\
W_{0}^{(m)} & =\frac{\lambda}{\left(n_{x}+\lambda\right)} \\
W_{0}^{(c)} & =W_{0}^{(m)}+\left(1-\alpha^{2}+\beta\right) \\
W_{i}^{(m)} & =\frac{1}{2 \cdot(n+\lambda)} \\
\lambda & =\alpha^{2}\left(n_{x}+\kappa\right)-n_{x}
\end{aligned}
$$

where $n_{x}$ is the dimension of $x ; \bar{x}$ and $P_{x}$ are the mean and covariance of $x$, respectively; $\kappa$ is the scaling parameter; and $\alpha, \beta$ are the control parameters.

Sigma points:

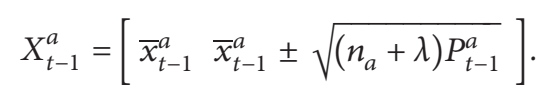

Time update:

$$
\left\{\begin{aligned}
X_{t \mid t-1}^{x} & =f\left(X_{t-1}^{x}, X_{t-1}^{v}\right), \\
\bar{x}_{t \mid t-1}= & \sum_{i=0}^{2 n_{a}} W_{i}^{(m)} X_{i, t \mid t-1}^{x}, \\
Y_{t \mid t-1}= & h\left(X_{t-1}^{x}, X_{t-1}^{n}\right), \\
\bar{y}_{t \mid t-1}= & \sum_{i=0}^{2 n_{a}} W_{i}^{(m)} Y_{i, t \mid t-1}^{x}, \\
P_{t \mid t-1}= & \sum_{i=0}^{2 n_{a}} W_{i}^{(c)}\left[X_{i, t \mid t-1}^{x}-\bar{x}_{t \mid t-1}\right]\left[X_{i, t \mid t-1}^{x}-\bar{x}_{t \mid t-1}\right]^{T},
\end{aligned}\right.
$$

where $Y_{i}=g\left(X_{i}\right)$ and $y=g(x)$ is a nonlinear transformation.

Measurement update: 


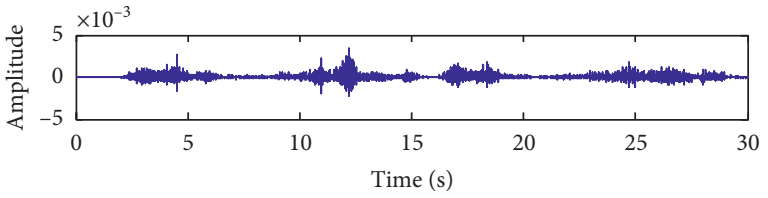

(a)

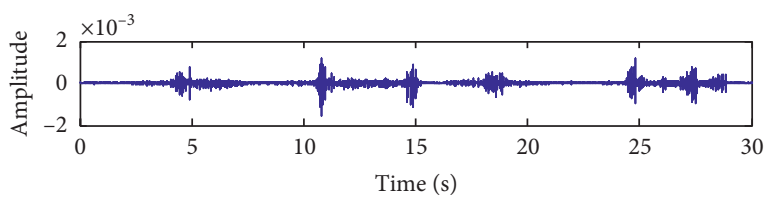

(c)

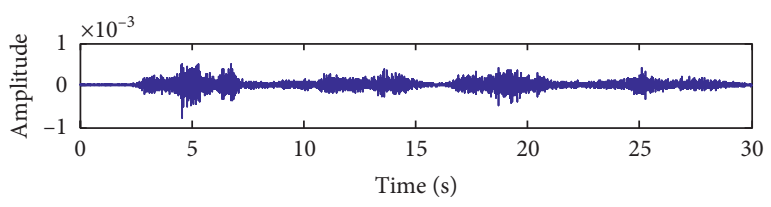

(e)

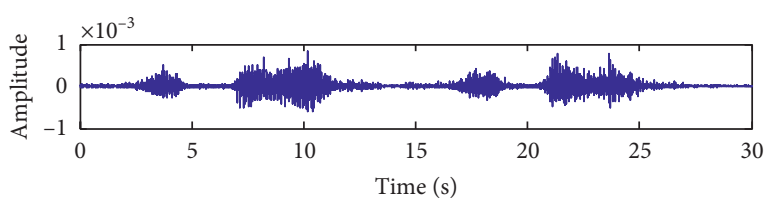

(g)

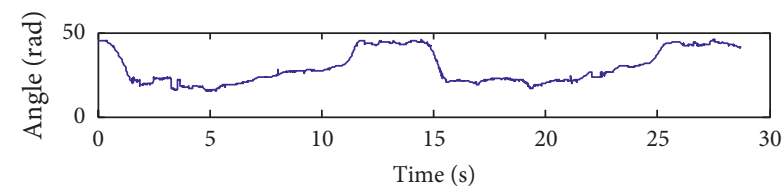

(i)

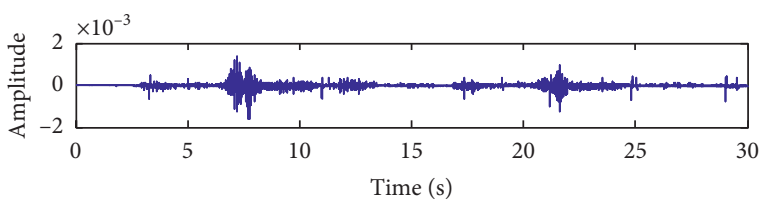

(b)

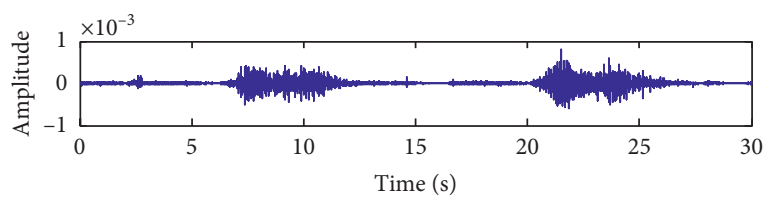

(d)

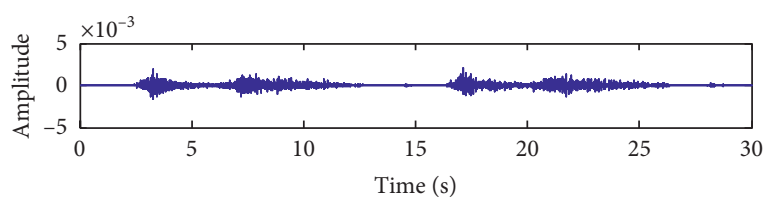

(f)

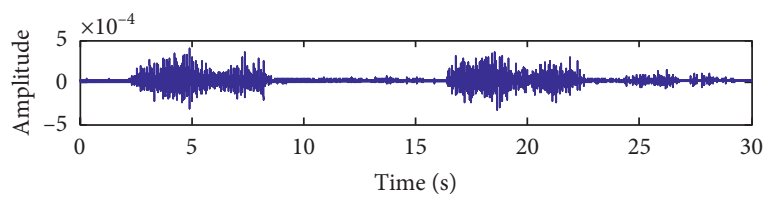

(h)

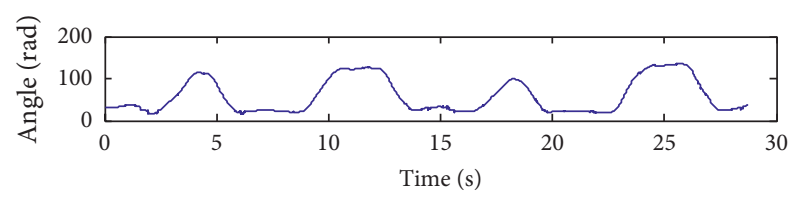

(j)

Figure 6: (a-h) sEMG signals from BB, TB, BR, TZ, TM, AD, LD, and PM. (i) Angle signal of shoulder joint. (j) Angle signal of elbow joint motion.

$$
\left\{\begin{array}{l}
P_{y_{t} y_{t}}=\sum_{i=0}^{2 n_{a}} W_{i}^{(c)}\left[Y_{i, t \mid t-1}-\bar{y}_{t \mid t-1}\right]\left[Y_{i, t \mid t-1}-\bar{y}_{t \mid t-1}\right]^{T} \\
P_{x_{t} y_{t}}=\sum_{i=0}^{2 n_{a}} W_{i}^{(c)}\left[X_{i, t \mid t-1}^{x}-\bar{x}_{t \mid t-1}\right]\left[Y_{i, t \mid t-1}^{x}-\bar{y}_{t \mid t-1}\right]^{T} \\
K_{t}=P_{x_{t} y_{t}} P_{y_{t} y_{t}}^{-1} \\
\bar{x}_{t}=\bar{x}_{t \mid t-1}+K_{t}\left(y_{t}-\bar{y}_{t \mid t-1}\right) \\
P_{t}=P_{t \mid t-1}-K_{t} P_{y_{t} y_{t}} K_{t}^{T}
\end{array}\right.
$$

Then, we substituted the variables of the nonlinear expression with the update equation through calculation and simulation.

\section{Experimental Results}

Ten subjects underwent two different situations, namely, loads of $3 \mathrm{~kg}$ and no load. Figure 6 shows the eight channels of the sEMG signals and joint motion recorded from a typical subject without load. Figure 7 depicts the eight channels of the MMG signals and joint motion recorded from a typical subject with load. Figure 8 illustrates the sEMG and MMG features from a typical subject.

Table 1 shows the identified values of the sEMG-MMG state-space model. The parameters are different in load and no-load conditions.

The identified parameters were substituted into the sEMG-MMG state-space model above to directly estimate the joint angle through the sEMG-MMG features and closed-loop prediction approaches.

The feature dataset of the multijoint movement was inputted into the proposed model. Five-fold cross validation was used to perform the simulation. Figure 9 shows the real and estimated joint angles from a typical subject. Table 2 lists the averaged results of the five subdatasets.

As shown in Figure 9 and Table 2, the mean RMSE and CC of the elbow joint with load are 5.27 and 0.96 , respectively, which indicates that the sEMG-MMG state-space model successfully kept the estimation convergent. 


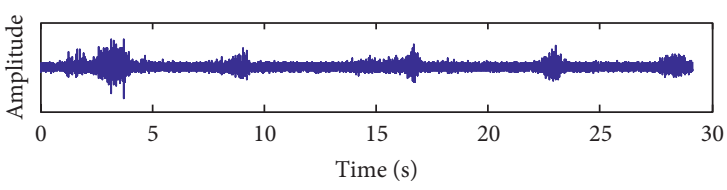

(a)

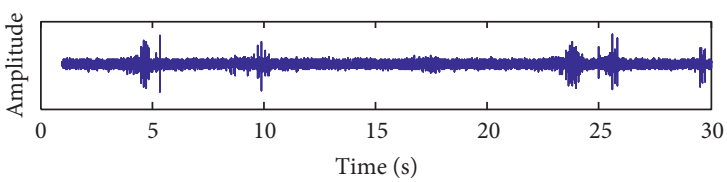

(c)

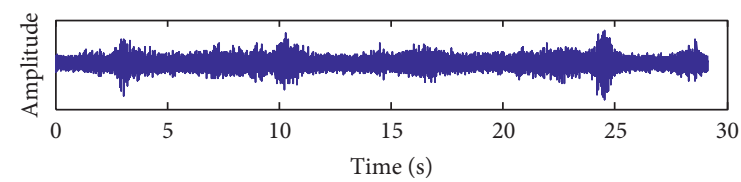

(e)

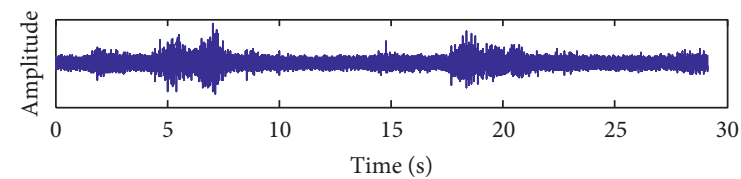

(g)

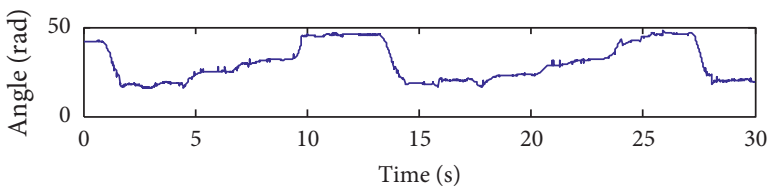

(i)

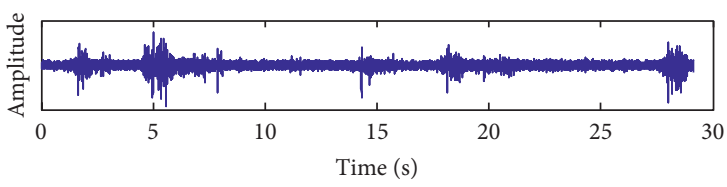

(b)

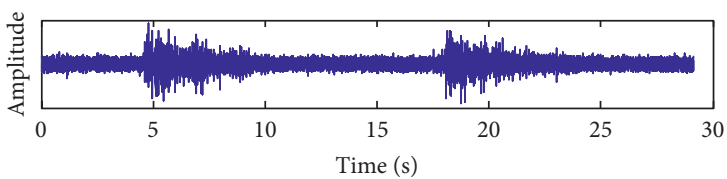

(d)

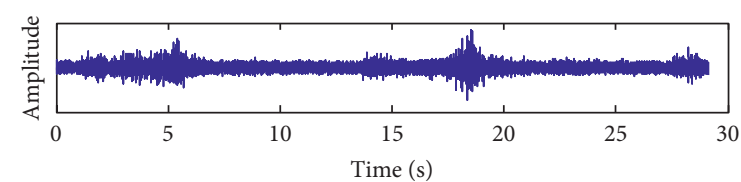

(f)

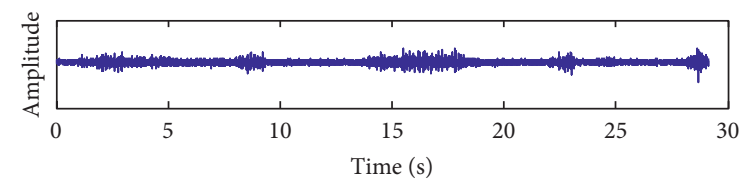

(h)

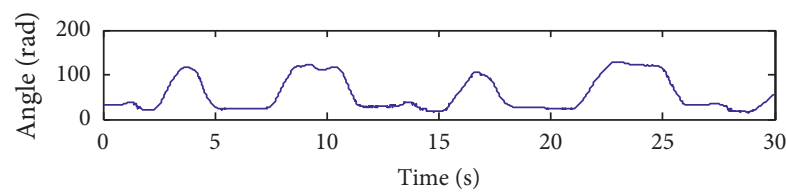

(j)

Figure 7: (a-h) MMG signals from BB, TB, BR, TZ, TM, AD, LD, and PM. (i) Angle signal of shoulder joint. (j) Angle signal of elbow joint motion.

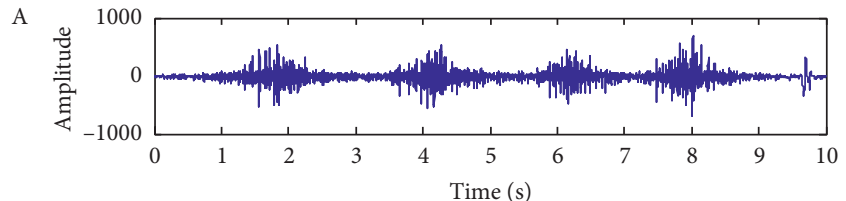

B

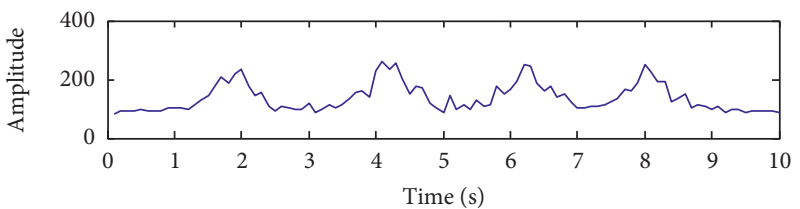

$\mathrm{C}$

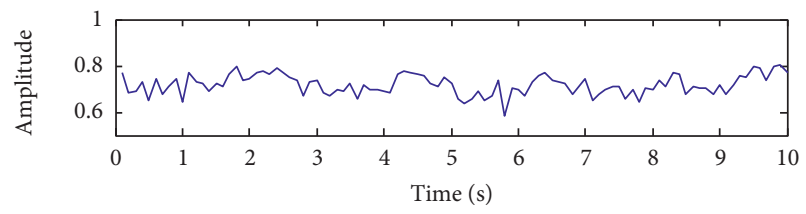

(a)

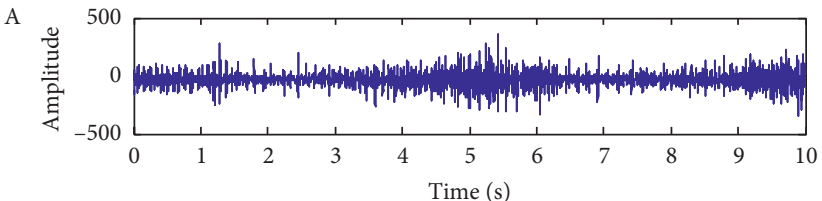

B
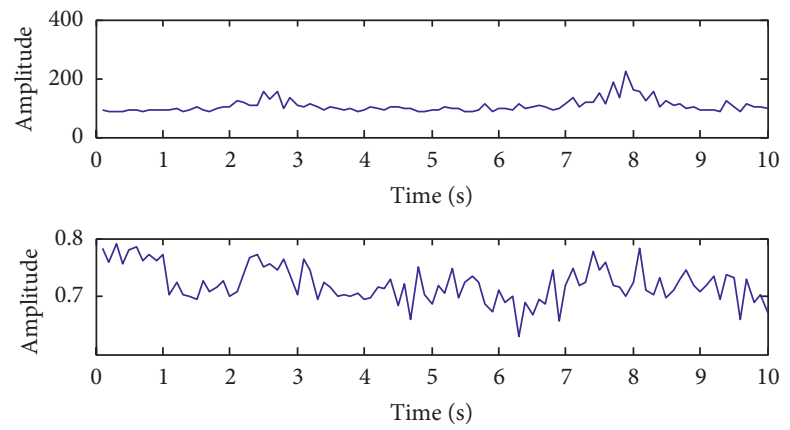

(b)

Figure 8: (a) sEMG signal from TM (A), WA (B), and PE (C). (b) MMG signals from AD (A), WA (B), and PE (C).

Moreover, the estimation errors of the elbow joint are smaller than those of the shoulder joint, and the load successfully improved the accuracy.
Five representative regression techniques, namely, the backpropagation neural network (BPNN) [29], generalized regression neural network (GRNN) [30], support vector 
TABLE 1: Identified parameters of the sEMG-MMG state-space model.

\begin{tabular}{|c|c|c|c|c|c|c|}
\hline$s_{i}$ & $s_{0}$ & $s_{1}$ & $s_{2}$ & $s_{3}$ & $s_{4}$ & $s_{5}$ \\
\hline Load & 5.26 & -8.57 & -5.37 & 0.02 & -0.13 & 0.58 \\
\hline No load & 1.88 & -1.97 & -0.56 & 0.08 & -0.68 & 0.24 \\
\hline$c_{i}^{1}$ & $c_{0}^{1}$ & $c_{1}^{1}$ & $c_{2}^{1}$ & $c_{3}^{1}$ & $c_{4}^{1}$ & $c_{5}^{1}$ \\
\hline Load & -2.67 & -0.07 & 4.43 & -0.24 & -1.59 & 0.02 \\
\hline No load & -0.39 & 0.01 & 1.57 & 0.12 & -0.29 & 0.24 \\
\hline$c_{i}^{2}$ & $c_{0}^{2}$ & $c_{1}^{2}$ & $c_{2}^{2}$ & $c_{3}^{2}$ & $c_{4}^{2}$ & $c_{5}^{2}$ \\
\hline Load & -6.43 & 0.22 & 8.35 & 0.03 & -2.54 & -0.04 \\
\hline No load & -2.34 & 0.23 & 8.69 & 0.48 & -2.41 & -0.15 \\
\hline
\end{tabular}

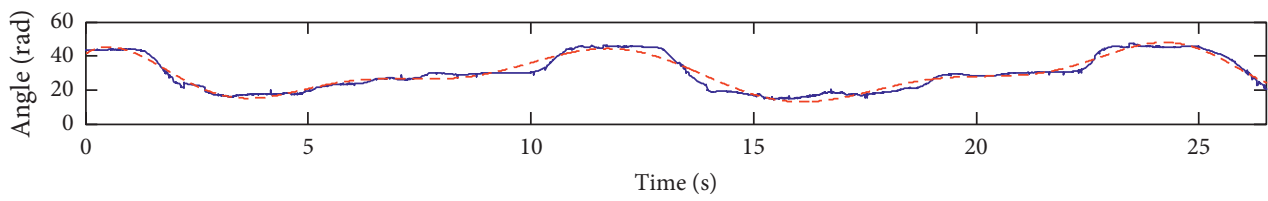

(a)

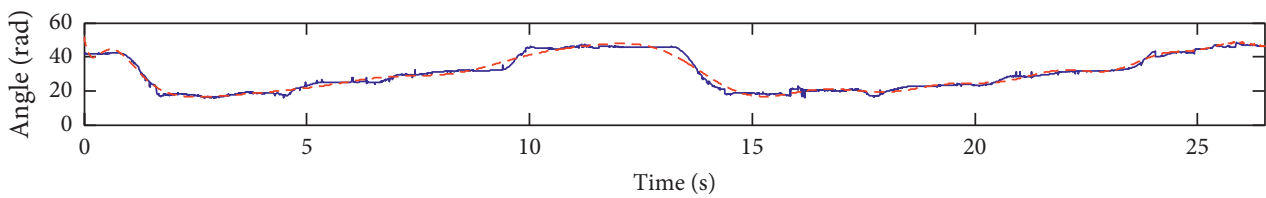

(b)

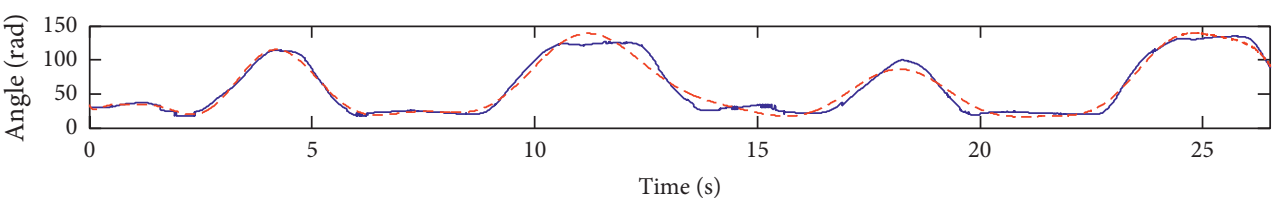

(c)

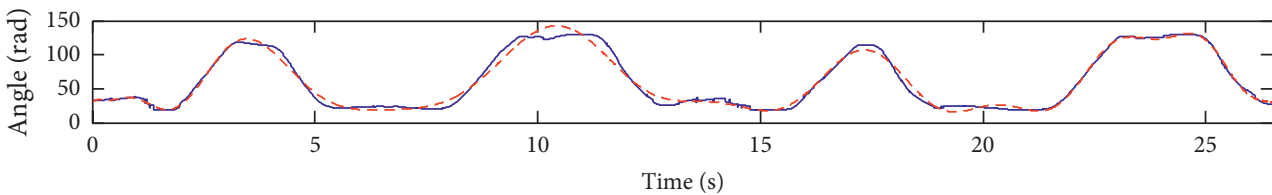

(d)

Figure 9: (a) Real and estimated shoulder joint angles without load. (b) Real and estimated shoulder joint angles with load. (c) Real and estimated elbow joint angles without load. (d) Real and estimated elbow joint angle with load. Red indicates estimated value; blue indicates Codamotion measurement.

TABLE 2: Evaluation of the performance of the proposed model (RMSE: root mean square error, CC: correlation coefficient).

\begin{tabular}{lcc}
\hline & RMSE & CC \\
\hline Elbow joint without load & $5.86 \pm 2.32$ & $0.95 \pm 0.02$ \\
Elbow joint with load & $5.27 \pm 2.03$ & $0.96 \pm 0.02$ \\
Shoulder joint without load & $7.89 \pm 1.68$ & $0.92 \pm 0.03$ \\
Shoulder joint with load & $7.26 \pm 1.73$ & $0.93 \pm 0.02$ \\
\hline
\end{tabular}

regression (SVR) [31], Fisher linear discriminant analysis (FDA) [32], and fuzzy min-max neural network (FMMNN) [33] were compared to verify the effectiveness of the proposed model. The same sEMG and MMG signals and features (WA and PE) and training set of neural networks were used in the experiments. The results are shown in Figure 10 and Table 3.
As shown in Figure 10 and Table 3, the proposed model exhibits the best performance in the experiments. The mean RMSE and CC of the proposed model are 6.27 and 0.95, respectively. The errors of the proposed model are much smaller than those of the other regression methods.

\section{Discussion}

This study aimed to build an sEMG-MMG state-space model to accurately estimate continuous joint movements from sEMG signals. The results showed that the proposed model improved the estimation accuracy of elbow-joint and shoulder-joint motions, with mean RMSEs of less than 5.3 and 7.3, respectively, indicating that the sEMG-MMG state-space model successfully kept the estimation convergent. Moreover, the proposed model performed better 


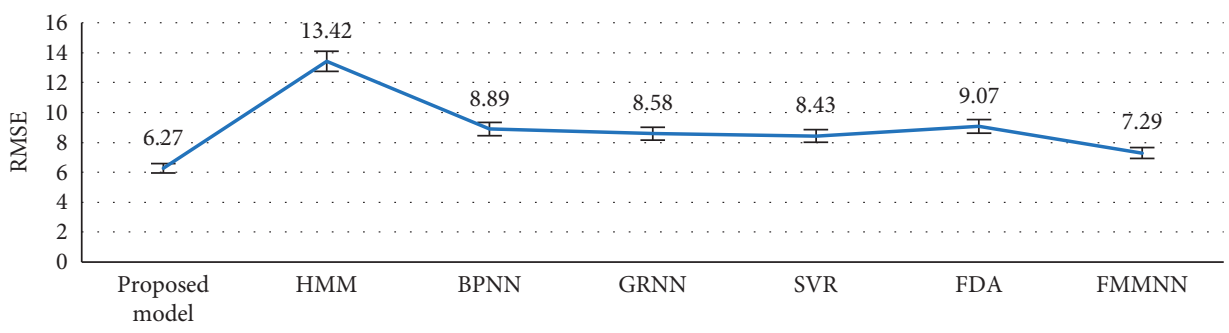

(a)

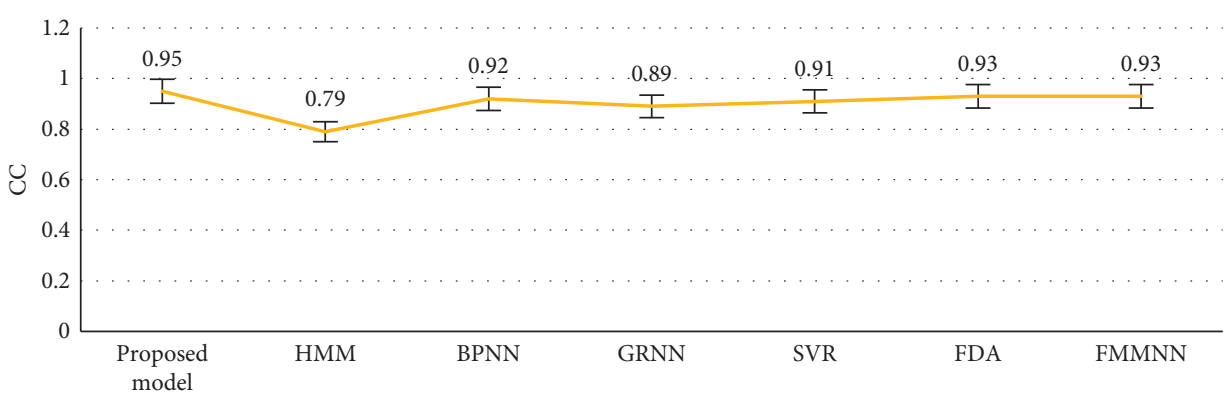

(b)

FIgURE 10: Mean RMSE and CC of seven regression models.

TABle 3: Evaluation of the performance of the proposed model (RMSE: root mean square error, CC: correlation coefficient).

\begin{tabular}{lcc}
\hline & RMSE & CC \\
\hline Proposed model & $6.27 \pm 0.63$ & $0.95 \pm 0.02$ \\
HMM & $13.42 \pm 2.59$ & $0.79 \pm 0.09$ \\
BPNN & $8.89 \pm 1.03$ & $0.92 \pm 0.03$ \\
GRNN & $8.58 \pm 0.73$ & $0.89 \pm 0.04$ \\
SVR & $8.43 \pm 0.82$ & $0.91 \pm 0.05$ \\
FDA & $9.07 \pm 1.03$ & $0.93 \pm 0.02$ \\
FMMNN & $7.29 \pm 0.78$ & $0.93 \pm 0.01$ \\
\hline
\end{tabular}

in the experiments than the conventional regression methods. In the comparative experiment, all of these methods were limited by data size, causing the neural networks to perform poorer than the sEMG-MMG statespace model because the training of neural networks requires more data sets.

Several basic limitations were associated with this study that need further development to be used in rehabilitation robots under real-world conditions. sEMG and MMG signals with specific motions were collected from healthy volunteers in a laboratory environment. Therefore, the performance of this model in real scenario with unscripted free-form activities performed by elderly or actual patients is unknown. Although no considerable difference in the inherent characteristics of the sEMG and MMG signals was found between subjects with and without disabilities, the amplitude and frequency of the signal still influenced the estimation accuracy. In this study, each individual was trained separately and required multiple repetitions of the task to obtain sufficient data for training and testing purposes. However, its difference from joint motions in real life with a larger task set is unclear. Also, the sEMG-MMG state-space model ignores some complex physiological parameters of HMM, which can result in prediction errors. These conditions need to be investigated before using the proposed model for clinical purposes.

\section{Conclusions}

In this study, a sEMG-MMG state-space model for multijoint motion estimation was constructed, where the continuous angular displacement could be estimated using the sEMG and MMG signals. Extensive experiments and comparison between the estimations and Codamotion measurements demonstrated the effectiveness of the proposed model. Compared with traditional methods for angle estimation, the proposed technique improved the estimation accuracy and real-time characteristics. In the future, the proposed method could be utilized for the training of rehabilitation robots and exoskeleton robots.

\section{Data Availability}

The data used to support the findings of this study are available from the corresponding author upon request.

\section{Conflicts of Interest}

The authors declare that they have no conflicts of interest.

\section{Acknowledgments}

This work was supported by the National Natural Science Foundation of China (nos. 61971169, 61671197, 61673350, and 60903084), and Jinhua Science and Technology Research Project (no. 2019-3-020). 


\section{References}

[1] J. L. Pons, Wearable Robots: Biomechatronic Exoskeletons, John Wiley \& Sons, Hoboken, NJ, USA, 2008.

[2] A. Cutti, P. Perego, M. Fusca, R. Sacchetti, and G. Andreoni, "Assessment of lower limb prosthesis through wearable sensors and thermography," Sensors, vol. 14, no. 3, pp. 5041-5055, 2014.

[3] J. L. G. Nielsen, S. Holmgaard, N. Ning Jiang, K. B. Englehart, D. Farina, and P. A. Parker, "Simultaneous and proportional force estimation for multifunction myoelectric prostheses using mirrored bilateral training," IEEE Transactions on Biomedical Engineering, vol. 58, no. 3, pp. 681-688, 2011.

[4] J. Liu and P. Zhou, "A novel myoelectric pattern recognition strategy for hand function restoration after incomplete cervical spinal cord injury," IEEE Transactions on Neural Systems and Rehabilitation Engineering, vol. 21, no. 1, pp. 96-103, 2013.

[5] O. Van Der Niet Otr, H. A. Reinders-Messelink, R. M. Bongers, H. Bouwsema, and C. K. Van Der Sluis, "The i-LIMB hand and the DMC plus hand compared: a case report," Prosthetics and Orthotics International, vol. 34, no. 2, pp. 216-220, 2010.

[6] B. Cesqui, P. Tropea, S. Micera, and H. Krebs, "EMG-based pattern recognition approach in post stroke robot-aided rehabilitation: a feasibility study," Journal of Neuroengineering and Rehabilitation, vol. 10, no. 1, p. 75, 2013.

[7] J. Silva, W. Heim, and T. Chau, "A self-contained, mechanomyography-driven externally powered prosthesis," Archives of Physical Medicine and Rehabilitation, vol. 86, no. 10, pp. 2066-2070, 2005.

[8] H.-B. Xie, Y.-P. Zheng, and J.-Y. Guo, "Classification of the mechanomyogram signal using a wavelet packet transform and singular value decomposition for multifunction prosthesis control," Physiological Measurement, vol. 30, no. 5, pp. 441-457, 2009.

[9] Y. Geng, L. Chen, L. Tian, and G. Li, "Comparison of electromyography and mechanomyogram in control of prosthetic system in multiple limb positions," in Proceedings of the 2012 IEEE-EMBS International Conference on Biomedical and Health Informatics, pp. 788-791, Hong Kong, China, January 2012.

[10] N. Alves and T. Chau, "Uncovering patterns of forearm muscle activity using multi-channel mechanomyography," Journal of Electromyography and Kinesiology, vol. 20, no. 5, pp. 777-786, 2010.

[11] N. Alves and T. Chau, "Recognition of forearm muscle activity by continuous classification of multi-site mechanomyogram signals," in Proceedings of the 2010 Annual International Conference of the IEEE Engineering in Medicine and Biology, pp. 3531-3534, IEEE, Buenos Aires, Argentina, September 2010.

[12] S. Sasidhar, S. K. Panda, and J. Xu, "A wavelet feature based mechanomyography classification system for a wearable rehabilitation system for the elderly," in Inclusive Society: Health and Wellbeing in the Community, and Care at Home, J. Biswas, H. Kobayashi, L. Wong, B. Abdulrazak, and M. Mokhtari, Eds., vol. 7910, pp. 45-52, Springer, Berlin, Germany, 2013.

[13] N. Alves and T. Chau, "Classification of the mechanomyogram: its potential as a multifunction access pathway," in Proceedings of the 2009 Annual International Conference of the IEEE Engineering in Medicine and Biology Society, pp. 29512954, IEEE, Minneapolis, MN, USA, September 2009.

[14] F. Zhang, P. Li, Z.-G. Hou et al., "sEMG-based continuous estimation of joint angles of human legs by using BP neural network," Neurocomputing, vol. 78, no. 1, pp. 139-148, 2012.
[15] Y. M. Aung and A. Al-Jumaily, "Estimation of upper limb joint angle using surface EMG signal," International Journal of Advanced Robotic Systems, vol. 10, no. 10, p. 369, 2013.

[16] T. S. Buchanan, D. G. Lloyd, K. Manal, and T. F. Besier, "Neuromusculoskeletal modeling: estimation of muscle forces and joint moments and movements from measurements of neural command," Journal of Applied Biomechanics, vol. 20, no. 4, pp. 367-395, 2004.

[17] C. Fleischer and G. Hommel, "A human-exoskeleton interface utilizing electromyography," IEEE Transactions on Robotics, vol. 24, no. 4, pp. 872-882, 2008.

[18] D. G. Lloyd and T. F. Besier, "An EMG-driven musculoskeletal model to estimate muscle forces and knee joint moments in vivo," Journal of Biomechanics, vol. 36, no. 6, pp. 765-776, 2003.

[19] R. D. Woittiez, P. A. Huijing, H. B. K. Boom, and R. H. Rozendal, "A three-dimensional muscle model: a quantified relation between form and function of skeletal muscles," Journal of Morphology, vol. 182, no. 1, pp. 95-113, 1984.

[20] Q. C. Ding, A. B. Xiong, X. G. Zhao, and J. D. Han, "A novel EMG-driven state space model for the estimation of continuous joint movements," in Proceedings of the 2011 IEEE International Conference on Systems, Man, and Cybernetics, pp. 2891-2897, Anchorage, AK, USA, October 2011.

[21] F. E. Zajac, "Muscle and tendon: properties, models, scaling and application to biomechanics and motor control," Critical Reviews in Biomedical Engineering, vol. 17, no. 4, pp. 359-411, 1989.

[22] K. R. S. Holzbaur, W. M. Murray, and S. L. Delp, "A model of the upper extremity for simulating musculoskeletal surgery and analyzing neuromuscular control," Annals of Biomedical Engineering, vol. 33, no. 6, pp. 829-840, Jun. 2005.

[23] P. Pigeon, L. H. Yahia, and A. G. Feldman, "Moment arms and lengths of human upper limb muscles as functions of joint angles," Journal of Biomechanics, vol. 29, no. 10, pp. 1365-1370, 1996.

[24] J. W. Ramsay, B. V. Hunter, and R. V. Gonzalez, "Muscle moment arm and normalized moment contributions as reference data for musculoskeletal elbow and wrist joint models," Journal of Biomechanics, vol. 42, no. 4, pp. 463-473, 2009.

[25] S. H. Park and S. P. Lee, "EMG pattern recognition based on artificial intelligence techniques," IEEE Transactions on Rehabilitation Engineering, vol. 6, no. 4, pp. 400-405, 1998.

[26] E. Olofsen, J. W. Sleigh, and A. Dahan, "Permutation entropy of the electroencephalogram: a measure of anaesthetic drug effect," British Journal of Anaesthesia, vol. 101, no. 6, pp. 810-821, 2008.

[27] C. Bandt and B. Pompe, "Permutation entropy: a natural complexity measure for time series," Physical Review Letters, vol. 88, no. 17, p. 174102, 2002.

[28] R. Merwe, A. Doucet, N. Freitas, and E. Wan, The Unscented Particle Filter, Technical Report CUED/F-INFENG/TR 380, Cambridge University Engineering Department, Cambridge, UK, 2000.

[29] P. D. Heermann and N. Khazenie, "Classification of multispectral remote sensing data using a back-propagation neural network," IEEE Transactions on Geoscience and Remote Sensing, vol. 30, no. 1, pp. 81-88, 1992.

[30] D. F. Specht, "A general regression neural network," IEEE Transactions on Neural Networks, vol. 2, no. 6, pp. 568-576, 1991.

[31] Q. L. Li, Y. Song, and Z. G. Hou, "Estimation of lower limb periodic motions from sEMG using least squares support vector regression," Neural Processing Letters, vol. 41, no. 3, pp. 371-388, 2015. 
[32] A. R. Webb, Statistical Pattern Recognition, John Wiley \& Sons, Hoboken, NJ, USA, 2002.

[33] M. F. Mohammed, C. P. Lim, and A. Quteishat, "A novel trust measurement method based on certified belief in strength for a multi-agent classifier system," Neural Computing and Applications, vol. 24, no. 2, pp. 421-429, 2014. 